\title{
Analogue versus propositional representation in congenitally blind individuals
}

\author{
PIERS FLEMING, LINDEN J. BALL, THOMAS C. ORMEROD, and ALAN F. COLLINS \\ Lancaster University, Lancaster, England
}

\begin{abstract}
Congenitally blind individuals are generally less accurate at mentally manipulating objects than sighted people. However, they often score higher on tests of short- and long-term verbal memory, and it has been suggested that an enhanced propositional representation compensates for inefficiencies in analogue visuospatial representation. Here, congenitally blind, blindfolded, and sighted participants recalled descriptions of relative object locations. In contrast to previous findings, the congenitally blind participants were as accurate as the blindfolded and sighted individuals at remembering the relative locations of objects, but their memory for the verbatim structure of presented descriptions was worse. We propose that, like sighted people, the congenitally blind spontaneously construct and remember analogue representations of object locations and that the performance discrepancies of the blind arise from the process of managing and manipulating these analogue representations.
\end{abstract}

How humans mentally represent spatial information remains controversial. Some researchers hold that spatial information is represented analogically (Kosslyn, 1994), whereas others claim that it is represented by a propositional grammar (Pylyshyn, 2002). However, discriminating between these representations is difficult, as even recent neuroscience research has demonstrated (Pylyshyn, 2003). One claim is that the underlying functional and anatomical mechanisms for both imagery and perception are very similar (Kosslyn, 1994). This view is supported by findings from unilateral spatial neglect patients whose deficits on remembered images mirror their perceptual neglect (Bisiach \& Luzzatti, 1978). Kosslyn, Thompson, Kim, and Alpert (1995) also found evidence of visual area activation with mental imagery. However, more recent findings of double dissociations make it untenable to claim identical imagery and perceptual systems, although a fair degree of overlap seems likely (see Bartolomeo, 2002 , for a review).

Behavioral data are also inconclusive, but there is increasing evidence that sighted people construct analogue representations to mimic structural relations between objects in the external world (Zwaan \& Radvansky, 1998). Mani and Johnson-Laird (1982) devised a subtle experimental paradigm that measured participants' ability to recognize verbatim, gist, and foil descriptions of spatial locations as indicators of propositional and analogue representational dominance. In this article, we use this para-

We gratefully acknowledge the Royal National Institute for the Blind for providing a research grant to T.C.O. and for assisting in the recruitment of participants. We thank Cathleen Moore, Thomas Baguley, and two anonymous reviewers for their helpful comments on an earlier version of this article. Correspondence should be addressed to L. J. Ball, Department of Psychology, Lancaster University, Lancaster LA1 4YF, England (e-mail: 1.ball@lancaster.ac.uk). digm to examine the representational characteristics of spatial reasoning among the congenitally blind.

In the absence of visual experience, one might expect congenitally blind individuals to derive representations of space that are profoundly different from those of sighted individuals. Perception based on haptic or auditory information alone might promote nonanalogue representation that would be suboptimal for spatial tasks. This argument has been used to explain slower performance by congenitally blind participants on mental rotation tasks (Marmor \& $\mathrm{Za}$ back, 1976). Nonanalogue representations may also provide a compensatory strategy for visuospatial memory; Vanlierde and Wanet-Defalque (2004) found no differences in blind and sighted individuals' memory for verbal descriptions of 2-D matrix patterns, with blind participants reporting use of a coordinate strategy to remember patterns. This strategy could be as effective as mental imagery because the blind may have superior verbal short- and long-term memory abilities (Hull \& Mason, 1995; Röder, Rösler, \& Neville, 2001), reflecting apparent use of the visual cortex for verbal tasks (Amedi, Raz, Pianka, Malach, \& Zohary, 2003).

However, the congenitally blind may use analogue representations. Perceptual mechanisms, such as those underpinning object recognition, appear to be cross modal (Amedi, von Kriegstein, van Atteveldt, Beauchamp, \& Naumer, 2005); it is possible to have spatial images without visual content (Jeannerod \& Jacob, 2005), and the blind have enhanced auditory-spatial abilities (Röder et al., 1999). One view of blind individuals' analogue representations is that they might differ from those of the sighted because the blind lack adequate frame-ofreference cues that aid performance on tasks like mental rotation (Heller, 1989). Recent research suggests that allocentric knowledge plays a role in spatial memory (Burgess, Spiers, \& Paleologou, 2004) and that frame of reference differs qualitatively depending on early visual 
experience (Röder, Rösler, \& Spence, 2004). Millar has long suggested (e.g., 1994) that the paucity of allocentric knowledge is responsible for poorer performance by the blind in visuospatial tasks.

In contrast, Vecchi (1998) argued that analogue representations used by sighted and blind people are equivalent, but the processing associated with such representations is less efficient in the case of blind people. A processing efficiency view holds that visuospatial working memory comprises a passive storage component (i.e., some form of representation), which underpins the recall of previously memorized material, and an active processing component by which representations are constructed, updated, and manipulated (cf. Logie, 1995). Blind individuals are viewed as having more limited abilities in the active component in comparison with the sighted, but equal abilities in the passive maintenance of encoded representations. In support of these proposals, blind participants recall actively constructed pathways less accurately than sighted participants but are equally accurate in recalling marked points within a matrix (Vecchi, 1998). However, evidence for this processing efficiency view has been inconclusive; Aleman, van Lee, Mantione, Verkoijen, and de Haan (2001), for example, observed no differential effect of spatial interference (in a tapping task) on blind and sighted groups and found an equivalent performance decrement on both active and passive tasks.

A strength of the processing efficiency view is that it explains evidence that patterns of performance by blind and sighted individuals are actually very similar on many visuospatial tasks. Blind participants demonstrate the characteristic linear relation between time and distance shown by sighted controls in mental rotation (Marmor \& Zaback, 1976) and mental scanning (Kerr, 1983), albeit at slower rates overall. The blind, like the sighted, are better at remembering concrete (i.e., imageable) words than abstract words (Zimler \& Keenan, 1983). Landau (1991) showed that a blind child performed similarly to sighted controls in representing and transforming objects. Heller et al. (2002) found that the Müller-Lyer illusion is equally compelling in the blind and the sighted. Gregory (2004) showed that a previously blind man, able to see for the first time, could read block capitals that he had learned to read by touch, and Intraub (2004) found the same boundary extension when remembering a scene in a blind person as in sighted people. The latter is possible because of the multimodal nature of spatial representations.

These previous tasks are all traditionally viewed as requiring analogue representations; they could be carried out using nonanalogue information, but unless the representations were so equivalent as to be indistinguishable, then very different patterns of performance would be expected. Overall, this corpus of evidence suggests that the blind can make use of analogue representations.

In the present study we investigated blind and sighted people's memory for short descriptions of relative spatial relations among objects, exemplified in Figure 1. Researchers (e.g., Baguley \& Payne, 2000; Mani \& JohnsonLaird, 1982) have used this task to determine the roles of analogue and propositional representations in spatial reasoning by assessing recognition (among foils) of verbatim descriptions (those that replicate the exact verbal form of the originally presented information) and gist descriptions (those that capture the meaning of verbal descriptions in different words).

Studies employing this paradigm have shown that sighted participants judge gist descriptions as being identical to the originally presented descriptions, suggesting conversion of the verbal descriptions into analogue representations that fit both versions. Furthermore, Mani and Johnson-Laird (1982) found that people recognize gist descriptions more effectively when spatial relations are determinate (i.e., the description allows only one possible layout) than when they are indeterminate (i.e., the description allows for more than one possible layout, thereby necessitating multiple analogue representations to capture all layout possibilities). If participants store and manipulate the spatial description in a propositional representation, then determinate and indeterminate layouts should be equally easy to remember. For example, the indeterminate relation "next to" and the determinate relation "to the left of" should be similar in their comprehensibility if represented in propositional form. However, the determinate relation can be represented analogically in a single scene, whereas the indeterminate relation brings to mind at least two possibilities and so would be harder to store in this format. The determinacy-indeterminacy distinction applies even in the case of recent formulations of analogue representations that suggest that they are not euclidean (i.e., exact analogues of perceived information) but are instead hierarchically organized (Huttenlocher, Hedges, Corrigan, \& Crawford, 2004).

The present experiment compared recognition of spatial location descriptions across three groups: congenitally blind, blindfolded, and sighted controls. The experimenter presented each description aurally, and participants then had to choose which of two layouts matched the description. The layouts were presented tactually, with object names being stated verbally by the experimenter when participants touched each location. This technique was employed in order to make the layouts accessible to the blind and blindfolded individuals. Participants then ranked four alternative versions of each description (verbatim, gist, and two foils) according to their degree of correspondence with the original description.

If blind participants rely upon propositional (i.e., nonanalogue) representations, then they should be more likely than sighted participants to treat gist descriptions as distractors. Moreover, they should perform the same with determinate and indeterminate relations. One might also expect blind participants to be more accurate in the recognition of verbatim descriptions than sighted participants because of their superior verbal memory (see, e.g., Röder et al., 2001). On the other hand, if blind participants are able to develop analogue representations of spatial descriptions, then they should show a pattern of performance identical to sighted participants' on the gist measure of recognition memory, and they should be as susceptible to the influence 


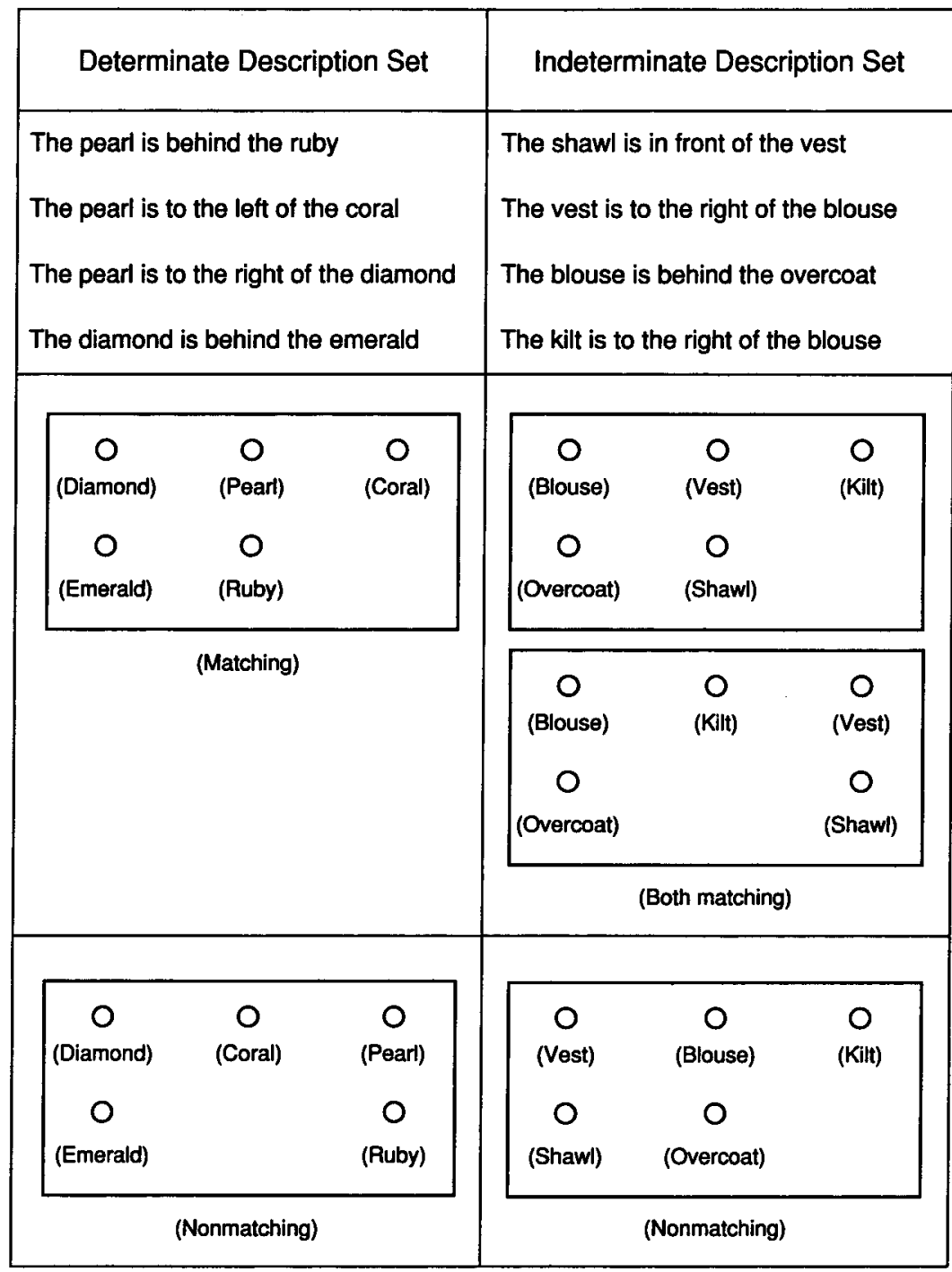

Figure 1. Examples of determinate and indeterminate description sets and corresponding diagrams. The determinate description set matches only a single diagram, whereas the indeterminate description set matches two diagrams (although only one would be presented as a matching diagram in the initial recognition task of the experiment). The circles represent tactile markers on the diagrams that were presented to participants.

of determinate versus indeterminate relations in their recognition of gist descriptions as sighted participants.

\section{METHOD}

\section{Participants}

The study involved 15 congenitally blind adults 1 (15-57 years old; mean, 38 years) and 32 adults from Lancaster University (19-29 years old; mean, 21 years). Blind and sighted individuals were matched for gender and were of a similar educational background and level. All participants were naive, paid volunteers. The blind participants were totally blind and had no memory of sight (see Table 1).

\section{Materials}

Eight sets of spatial descriptions and diagrams (plus two additional sets for practice trials) were produced using a stimulus gen- eration program developed by Baguley and Payne (2000). Each set contained four sentences describing spatial relations between five objects in a 2-D euclidean plane. Four description sets were determinate, each having only one possible object layout, and four sets were indeterminate, each having two possible object layouts. Each description set contained object names from a specific category (e.g., bird names) that were matched for frequency, imagery, and concreteness (Kučera \& Francis, 1967; Quinlan, 1992). Descriptions were counterbalanced for category name, configuration, and presentation order.

Matching and nonmatching tactile diagrams were produced for each description set (see Figure 1); the relative location of two objects described in the sentences was reversed in the nonmatching diagram. The tactile boards were $29 \times 38 \mathrm{~cm}$ plywood sheets with rubber stops (15-mm diameter projecting $10 \mathrm{~mm}$ from the plywood) representing object positions. The rubber stops were $15 \mathrm{~cm}$ apart. 
Table 1

Characteristics of Early-Blind Participants

\begin{tabular}{|c|c|c|c|c|}
\hline Sex & Age & $\begin{array}{l}\text { Years of Higher } \\
\text { Education }\end{array}$ & $\begin{array}{c}\text { Age at Onset of } \\
\text { Blindness }\end{array}$ & Etiology \\
\hline $\mathbf{M}$ & 55 & 0 & Birth & Retrolental fibroplasia \\
\hline $\mathbf{M}$ & 57 & 3.5 & Birth & Retrolental fibroplasia \\
\hline $\mathbf{M}$ & 51 & 5 & Birth & Retrolental fibroplasia \\
\hline $\mathbf{F}$ & 42 & 3 & 3 months -2 years & Bilateral retinoblastoma \\
\hline $\mathrm{F}$ & 48 & 5 & 1 year & Bilateral retinoblastoma \\
\hline $\mathbf{M}$ & 52 & 4 & Birth & Retrolental fibroplasia \\
\hline $\mathbf{M}$ & 54 & 10 & Birth & Congenital rubella \\
\hline $\mathbf{M}$ & 17 & 0 & Birth & Retrolental fibroplasia \\
\hline $\mathbf{M}$ & 16 & 0 & Birth & Retrolental fibroplasia \\
\hline $\mathbf{M}$ & 56 & $\mathbf{0}$ & Birth & Retrolental fibroplasia \\
\hline $\mathbf{M}$ & 16 & 0 & Birth & Retrolental fibroplasia \\
\hline $\mathbf{F}$ & 20 & 1 & Birth & Retrolental fibroplasia \\
\hline $\mathbf{F}$ & 15 & 0 & $3-4$ years & Bilateral retinoblastoma \\
\hline $\mathbf{M}$ & 50 & 4 & Birth & Retrolental fibroplasia \\
\hline $\mathbf{F}$ & 24 & 3 & Birth & Retrolental fibroplasia \\
\hline
\end{tabular}

Note-Retrolental fibroplasia (also termed retinopathy of prematurity) affects prematurely born babies, and is thought to be caused by disorganized growth of retinal blood vessels, resulting in scarring and retinal detachment. Oxygen toxicity may also contribute to its development. Retinoblastoma is a cancer of the retina that occurs mostly in children before the age of 5 years. The tumor may begin in one or both eyes.

For the description recognition task, four versions of each original description set were devised. One contained the original (verbatim) sentences. The second, a gist version, described the same object array as the original description but in different words. It shared one sentence with the original, with two paraphrased sentences (which inverted the objects and the spatial relations) and one inferable sentence that was true of, but not explicit in, the original description. The remaining versions were distractors that shared one sentence with the original description but swapped the relative locations of three objects.

\section{Procedure}

Each trial consisted of three phases: a presentation of the descriptions of object locations, a layout matching task, and an incidental recogniition task. The participants were not instructed to memorize descriptions but were instructed only to use them for the layout matching task (cf. Mani \& Johnson-Laird, 1982). The experimenter read the descriptions to the blindfolded and blind participants (the participants indicated when they were ready for the next sentence), whereas the sighted participants read descriptions themselves. In the layout matching phase, the participants examined two tactile boards, one that matched the description and one that did not; they were then required to identify the matching board. As the participants touched each rubber stop on the board, the object represented at that location was named by the experimenter. The participants received two practice trials followed by eight experimental trials. They received feedback on the practice trials only. The reading and layout matching phases were self-paced, and each took approximately $1 \mathrm{~min}$ per trial.

The final recognition phase began directly after completion of the eight layout matching tasks. For each recognition trial, the participants ranked, on a scale from 1-4, four versions of a description set (given in the first phase) according to how closely each version resembled the original description, ignoring the order of sentences within the description set (cf. Baguley \& Payne, 2000).

\section{RESULTS}

Errors accounted for $33 \%$ of the choices in the layout matching phase, a result that mirrors previous findings, such as those of Baguley and Payne (2000), who reported an error rate of $24 \%$. There were no reliable effects of group (congenitally blind, blindfolded, and sighted controls) $[F(2,44)=1.21, p=.31]$ or determinacy status (determinate vs. indeterminate descriptions) $[F(1,44)=$ $2.70, p=.11]$ on layout matching accuracy (Figure 2). Subsequent analyses excluded data from one blind and one blindfolded participant, who both scored at or below chance across all tasks.

Gist recognition (Figure 3), measured as the proportion of responses in which the two descriptions that described the correct layout (i.e., the verbatim and gist versions) were ranked first and second, was greater for determinate than for indeterminate relations $[F(1,42)=10.07, p<.05]$. However, there was neither an effect of group $(F<1)$ nor an interaction between group and determinacy status $(F<1)$.

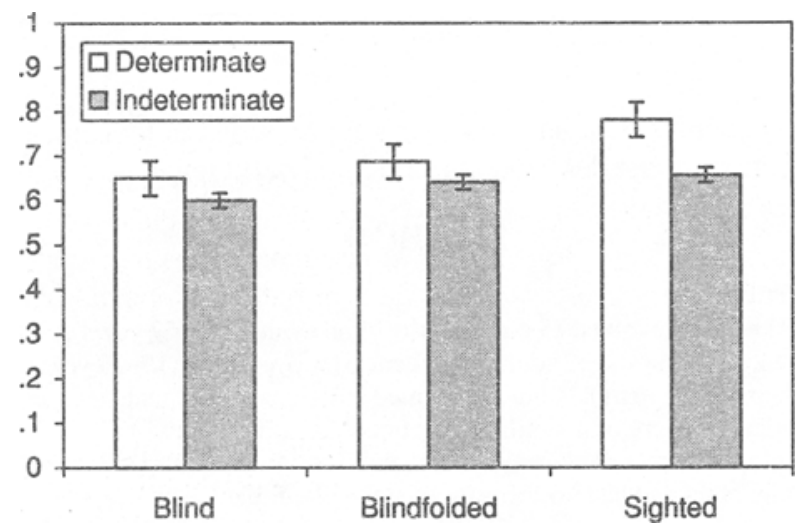

Figure 2. Proportion of correct judgments of the matching diagram by group for determinate and indeterminate description sets. Vertical lines depict standard errors of the means. 


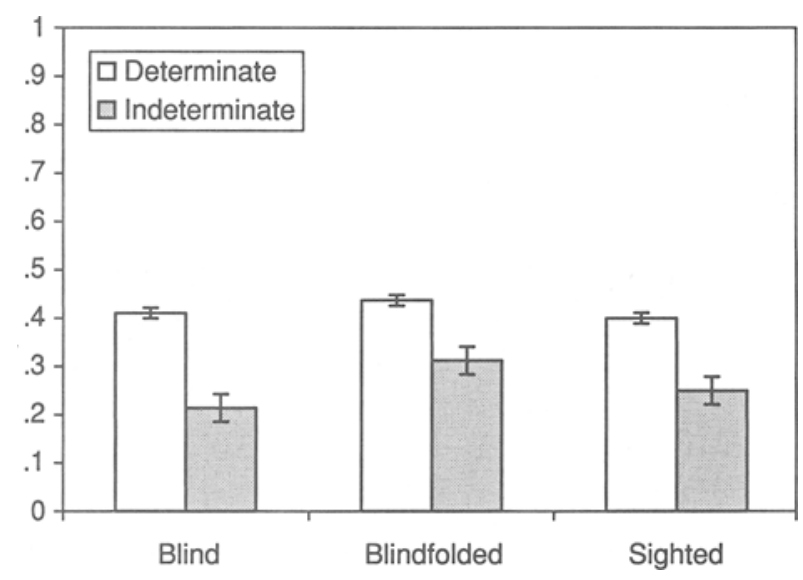

Figure 3. Proportion of correct gist ranking by group for determinate and indeterminate description sets. Vertical lines depict standard errors of the means.

Verbatim recognition (see Figure 4), measured as the proportion of responses in which the verbatim description was ranked first, differed significantly across groups $[F(2,42)=5.55, p<.01]$. Planned comparisons show that the congenitally blind group recognized fewer verbatim descriptions than the sighted groups $[F(1,42)=$ $7.95, p<.01]$. The difference between the sighted and blindfolded groups was not significant $[F(1,42)=2.97$, $p>.05]$. Performance on verbatim recognition contrasted starkly with digit spans: On this test, congenitally blind participants achieved scores between 6 and 14 items $(M=$ 8.64), compared with a range of between 6 and 8 items for sighted participants $(M=6.44)$, consistent with the normal range (Smyth \& Scholey, 1996).

One area of concern is the disparity in ages between the blind and the sighted participants. However, when the blind group was subdivided into those under 30 years old $(M=18)$ and those over 30 years old $(M=51)$, no differences were found between subgroups for gist performance $[t(12)=0.000, p=1]$, or for verbatim performance $[t(12)=0.149, p=.884]$.

\section{DISCUSSION}

In contrast to previous findings (see, e.g., Aleman et al., 2001), congenitally blind participants showed no decrement in performance of the visuospatial task relative to sighted and blindfolded participants. Similarly, there were no group differences in gist recognition performance, with superior gist recognition for determinate as opposed to indeterminate descriptions. Despite apparently above average digit spans (8.64 for blind vs. 6.44 for sighted), the verbatim recognition of congenitally blind participants was worse than that of sighted and blindfolded participants.

The key question is, how were congenitally blind participants able to match the spatial reasoning performance of blindfolded participants and sighted controls, when previous studies have shown a performance decrement among the congenitally blind on tasks that require the mental manipulation of objects? The answer appears to be that the blind use analogue representations, much as sighted people do. Their memory for object layouts was equivalent to that of sighted controls, a finding consistent with the use of analogue representations that are sensitive to determinacy and effective at retaining gist information, rather than abstract propositional representations that retain verbatim information only and are not sensitive to determinacy.

This equivalence of representations between sighted and blind groups is supported by recent research indicating that spatial knowledge can be acquired from nonvisual modalities (see, e.g., Amedi et al., 2005; Shelton \& McNamara, 2001). This suggests that the blind and the sighted construct and access cross-modal representations of objects and space. Vanlierde and Wanet-Defalque (2004) proposed that blind people's visuospatial performance reflects the use of a compensatory verbal strategy based on a coordinate model. However, gist recognition in the present study required the identification of nonexplicit locations that could not be inferred using a coordinate model without a performance decrement. Furthermore, verbatim recognition was, in fact, worse in the blind than in the sighted groups, a result that would be very unlikely if the blind relied on a verbal strategy. It seems likely that the blind can form representations that reflect their perceptions as the sighted do (Bartolomeo, 2002) and that the blind do not need to rely on a verbally based compensatory representation different from sighted representations.

An ancillary question is why congenitally blind participants performed worse than other groups in verbatim recognition, when studies of verbal memory ability in the blind suggest that the opposite should be the case (Hull \& Mason, 1995; Röder et al., 2001). We propose that the relatively poor verbatim performance of our blind participants is, paradoxically, unlikely to be the result of inherent deficiencies in verbal memory. Blind people tend to have equivalent (or better) verbal capabilities than sighted individuals. Our blind participants' poor verbatim recognition

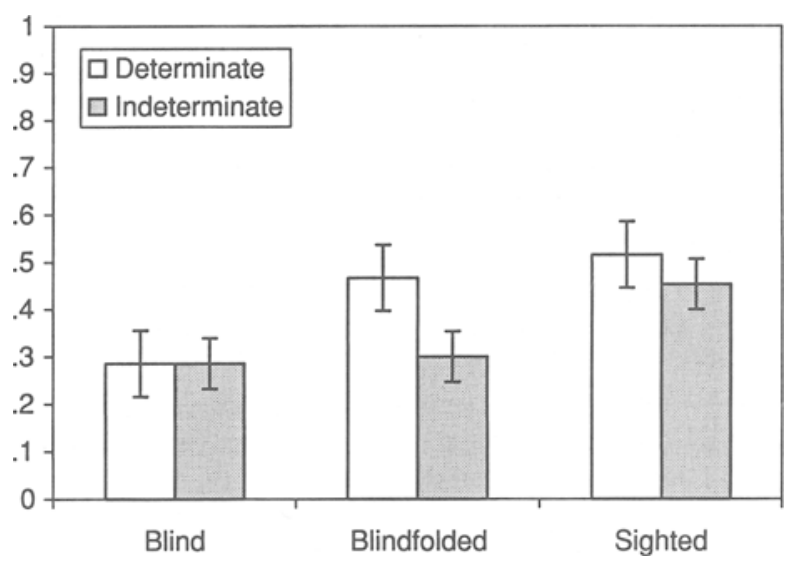

Figure 4. Proportion of correct verbatim ranking by group for determinate and indeterminate description sets. Vertical lines depict standard errors of the means. 
is also unlikely to result from the use of a verbal strategy or a lack of frame-of-reference information. Instead, we suggest that poorer verbal performance on visuospatial tasks by blind individuals may reflect the difficulty they have carrying out these tasks: Visuospatial tasks are simply more difficult for the congenitally blind.

Our proposal can be clarified as follows: Creating an analogue representation (i.e., a mental model) requires verbal working memory resources simply to retain the information and thus free up general working memory resources to construct the model, as evidenced by correlations between digit span and gist recognition (but not verbatim recognition) in our study ${ }^{2}$ (cf. Morra, 1989). Therefore, blind participants' superior verbal memory abilities may aid them in the creation of analogue models. In contrast, verbatim recognition is held to be a measure of the episodic trace of model construction processes (Baguley \& Payne, 2000). The blind are believed to be less effective at active processing (i.e., manipulation and construction) of spatial representations in the first place (Vecchi, 1998), which would offset their verbal span advantage and lead to a degraded episodic trace and poorer verbatim recognition performance. Previous research has indicated deficits in task performance by blind participants compared with that of sighted participants. Such poor task performance may, we argue, reflect a cost associated with the sheer cognitive burden of visuospatial processing.

In support of this latter view, we also note that research has shown that an increased cognitive burden in a visuospatial task can, indeed, lead to a specific decrement in verbatim memory. Barshi and Healy (2002) asked participants to repeat navigation instructions for an array that was perceived in either two or three dimensions, although the stated directions were informationally equivalent. It was found that participants' immediate verbal memory was far worse on the more complex 3-D matrix navigation task. This phenomenon mirrors that found in this experiment: The blind group had to do more to achieve the task and had worse verbatim recall.

Both our present findings and those of Barshi and Healy (2002) suggest that the supposedly verbal recall measure employed by researchers in the context of navigation tasks may not actually reflect a genuine measure of verbal memory, but rather may be an index of a propositional memory for the active processing that underpins the construction of analogue mental representations (Baguley \& Payne, 2000; Barshi \& Healy, 2002). Thus, although the blind are worse at spatial processing (which produced a decrement in verbal recognition in this study) than the sighted, they are still able to build analogue representations and, having built them, are effective at maintaining them.

In summary, the present study provides support for the view that the sighted and the blind can make similar use of analogue representations when tackling visuospatial tasks. The consistent performance deficits observed in the blind on such tasks are not necessarily the result of the deployment of different representations, but instead may reflect differences in processing effectiveness.

\section{REFERENCES}

Aleman, A., van Lee, L., Mantione, M. H. M., Verkoijen, I. G., \& DE HAAN, E. H. F. (2001). Visual imagery without visual experience: Evidence from congenitally totally blind people. NeuroReport, 12, 2601-2604.

Amedi, A., Raz, N., Pianka, P., Malach, R., \& Zohary, E. (2003). Early "visual" cortex activation correlates with superior verbal memory performance in the blind. Nature Neuroscience, 6, 758-766.

Amedi, A., von Kriegstein, K., van ATteVeldt, N. M., Beauchamp, M. S., \& NAUMER, M. J. (2005). Functional imaging of human crossmodal identification and object recognition. Experimental Brain Research, 166, 559-571.

BAGULEY, T., \& PAYNe, S. J. (2000). Long-term memory for spatial and temporal mental models includes construction processes and model structure. Quarterly Journal of Experimental Psychology, 53A, 479. 512.

BARSHI, I., \& HEALY, A. F. (2002). The effects of mental representation on performance in a navigation task. Memory \& Cognition, 30, 1189-1203.

BARTOLOMEO, P. (2002). The relationship between visual perception and visual mental imagery: A reappraisal of the neuropsychological evidence. Cortex, 38, 357-378.

BisiACH, E., \& LUZZATTI, C. (1978). Unilateral neglect of representational space. Cortex, 14, 129-133.

Burgess, N., Spiers, H. J., \& Paleologou, E. (2004). Orientational manoeuvres in the dark: Dissociating allocentric and egocentric influences on spatial memory. Cognition, 94, 146-166.

GREGORY, R. (2004). The blind leading the sighted. Nature, 430, 836.

HELLER, M. A. (1989). Tactile memory in sighted and blind observers: The influence of orientation and rate of presentation. Perception, 18, 121-133.

Heller, M. A., Brackett, D. D., Wilson, K., Yoneyama, K., BOYER, A., \& STEFFEN, H. (2002). The haptic Müller-Lyer illusion in sighted and blind people. Perception, 31, 1263-1274.

Hull, T., \& MASON, H. (1995). Performance of blind children on digit span tests. Journal of Visual Impairment \& Blindness, 89, 166-169.

HutTenlocher, J., Hedges, L. V., Corrigan, B., \& Crawford, L. E. (2004). Spatial categories and the estimation of location. Cognition, 93, 75-97.

INTRAUB, H. (2004). Anticipatory spatial representation of 3D regions explored by sighted observers and a deaf-and-blind-observer. Cognition, 94, 19-37.

JEANNEROD, M., \& JACOB, P. (2005). Visual cognition: A new look at the two-visual systems model. Neuropsychologia, 43, 301-312.

KERR, N. H. (1983). The role of vision in "visual imagery" experiments: Evidence from the congenitally blind. Journal of Experimental Psychology: General, 112, 265-277.

KossLYN, S. M. (1994). Image and brain: The resolution of the imagery debate. Cambridge, MA: MIT Press.

Kosslyn, S. M., Thompson, W. L., KIM, I. J., \& AlPERT, N. M. (1995). Topographical representations of mental images in primary visual cortex. Nature, 378, 496-498.

KuČERA, H., \& FRANCIS, W. N. (1967). Computational analysis of presentday American English. Providence, RI: Brown University Press.

LANDAU, B. (1991). Spatial representation of objects in the young blind child. Cognition, 38, 145-178.

LOGIE, R. H. (1995). Visuo-spatial working memory. Hove, U.K.: Erlbaum.

MANI, K., \& JoHNSON-LAIRD, P. N. (1982). The mental representation of spatial descriptions. Memory \& Cognition, 10, 181-187.

MARMOR, G., \& ZABACK, L. (1976). Mental rotation by the blind: Does mental rotation depend on visual imagery? Journal of Experimental Psychology: Human Perception \& Performance, 2, 515-521.

MiLLAR, S. (1994). Understanding and representing space: Theory and evidence from studies with blind and sighted children. Oxford: Oxford University Press, Clarendon Press.

MORRA, S. (1989). Developmental differences in the use of verbatim versus spatial representations in the recall of spatial descriptions: A probabilistic model and an experimental analysis. Journal of Memory \& Language, 28, 37-55.

PYLYSHYN, Z. W. (2002). Mental imagery: In search of a theory. Behavioral \& Brain Sciences, 25, 157-238. 
PYLYShYN, Z. W. (2003). Return of the mental image: Are there really pictures in the brain? Trends in Cognitive Sciences, 7, 113-118.

QuinLAN, P. T. (1992). The Oxford psycholinguistic database. Oxford: Oxford University Press.

RÖDER, B., RöSLER, F., \& Neville, H. J. (2001). Auditory memory in congenitally blind adults: $A$ behavioral-electrophysiological investigation. Cognitive Brain Research, 11, 289-303.

RÖDER, B., RösLER, F., \& SPENCE, C. (2004). Early vision impairs tactile perception in the blind. Current Biology, 14, 121-124.

RöDER, B., TEDER-Sälejärvi, W., SterR, A., Rösler, F., Hillyard, S. A., \& NEVILLE, H. J. (1999). Improved auditory spatial tuning in blind humans. Nature, 400, 162-166.

Shelton, A. L., \& McNamara, T. P. (2001). Visual memories from nonvisual experiences. Psychological Science, 12, 343-347.

SMYTH, M. M., \& SchoLEY, K. A. (1996). The relationship between articulation time and memory performance in verbal and visuospatial tasks. British Journal of Psychology, 87, 179-191.

VANLIERDE, A., \& WANET-DEFALQUE, M. C. (2004). Abilities and strategies of blind and sighted subjects in visuo-spatial imagery. Acta Psychologica, 116, 205-222.

VECCHI, T. (1998). Visuo-spatial imagery in congenitally totally blind people. Memory, 6, 91-102.
ZiMLER, J., \& KeENAN, J. M. (1983). Imagery in the congenitally blind: How visual are visual images? Journal of Experimental Psychology: Learning, Memory, \& Cognition, 9, 269-282.

ZWAAN, R. A., \& RAdVANSKY, G. A. (1998). Situation models in language comprehension and memory. Psychological Bulletin, 123, 162185.

\section{NOTES}

1. We classified participants as congenitally blind based upon their self-reports, although we note that the medical histories of three individuals suggested that they might have had some early visual experience. Data reanalysis excluding these participants produced an identical pattern of results.

2. This is supported by the blind participants' data, which showed a trend for a correlation between digit span and gist recognition $(r=.303)$, but not between digit span and verbatim recognition $(r=.088)$.

(Manuscript received September 10, 2005; revision accepted for publication March 14, 2006.) 